\title{
Photometric Calibration of the CCD Camera of 1-m Telescope at VBO
}

\author{
Y. D. Mayya* Indian Institute of Astrophysics, Bangalore 560034
}

Received 1991 July 7; accepted 1991 October 10

\begin{abstract}
Calibration of the CCD camera of the 1-m telescope at the Vainu Bappu Observatory, Kavalur, to the $B V R$ system is reported here based on the observations of stars in the 'dipper asterism' in the open cluster M 67 (NGC 2682). Transformations involving $B$ and $V$ have negligible colour terms, while those involving $R$ are slightly colour dependent. The possibility of using scaled-down $R$ band fluxes to estimate the continuum flux at $\mathrm{H} \alpha$ is investigated by comparing the counts in $R$ band with those through an interference filter centred at $\mathrm{H} \alpha$. The scaling factor is found to remain constant over a wide range of colours. The sensitivity of the telescope-filter-CCD combination is estimated to be 2.0 per cent, 8.3 per cent and 9.7 per cent in $B, V$ and $R$ bands, respectively. The star F117 appears to be a small-amplitude ( $\sim 0.05 \mathrm{mag})$ variable.
\end{abstract}

Key words: CCD photometry-BVRH $\alpha$ photometry-atmospheric extinctionphotometric calibration-open clusters-M67 (NGC 2682)

\section{Introduction}

The CCD camera of the 1-m telescope at Vainu Bappu Observatory (VBO), Kavalur uses a Thomson CSF Th7882 CCD chip. The chip coated for enhanced ultraviolet sensitivity, mounted in a liquid-nitrogen-cooled dewar, together with the controller and the data acquisition system were acquired in an integrated form from Photometrics Ltd., USA. In the imaging mode the CCD is used at the $f / 13$ Cassegrain focus at a scale of 0.357 arcsec pixel ${ }^{-1}$ covering a total field of 137 arcsec $\times 206$ arcsec. An initial calibration of the system to UBVR system was done by Sagar \& Pati (1989) with Fernie (1974) filter combinations. We repeated the calibration using observations made on a better night in 1990 April. The colour coefficients for transformations involving $B$ and $V$ agreed with Sagar \& Pati (1989). However, the $B$ filter was found to be highly inefficient and the $R$ filter highly non-standard. Apart from the degradation of filters, the total thickness $(7 \mathrm{~mm})$ of the combination filter was responsible for the poor efficiency. A new system of filters agreeing closely with the set recommended by Bessell (1976) was hence used to replace the old filters in 1991. Table 1 lists the new filter combinations. The combined thickness of the different sets of filters is maintained the same $(4 \mathrm{~mm})$ to avoid focus differences. The glass combinations used for $B$ and $V$ are identical to those of Bessell (1976), while for $R$, RG 610 is used instead of OG 570 for lower wavelength cut-off following Schild (1983), since this was readily available. Our combination results in shifting of the effective wavelength for $R$ band

\footnotetext{
*Also Joint Astronomy Programme, Department of Physics, Indian Institute of Science, Bangalore 560012.
} 
Table 1. Glass filter combinations.

\begin{tabular}{ll}
\hline Band & Components (thickness in mm) \\
\hline$B$ & GG13(2) + BG12(1) + BG18(1) \\
$V$ & GG14(2) + BG18(2) \\
$R$ & RG610(2) + KG3(2) \\
\hline
\end{tabular}

slightly towards red, bringing it closer to $\mathrm{H} \alpha$. This is advantageous when one wishes to estimate the in-band continuum at $\mathrm{H} \alpha$ from $R$ band images.

The usefulness of the 'dipper asterism' field in M67 (NGC 2682) in calibrating CCDs for photometric purposes is discussed by several authors (Schild 1983; Sagar \& Pati 1989; Joner \& Tayler 1990; Chevalier \& Ilovaisky 1991). Gilliland et al. (1991) have carried out extensive $U B V R$ photometry of stars in the central region of the cluster. We have carried out the photometry of sixteen stars in this field. Ten of these are used to calibrate the system using the standard magnitudes of Chevalier \& Ilovaisky (1991). Details of observations are given in Section 2. In Section 3, data analysis methods, results on extinction, transformations and accuracies of photometry are discussed. Results are summarized in Section 4.

\section{Observations}

The observations were carried out during 1991 January-April, as part of $B V R H \alpha$ $\mathrm{CCD}$ photometry of extragalactic giant $\mathrm{H} \alpha \mathrm{II}$ regions. The dipper asterism field in M67 was observed regularly in order to obtain accurate transformation coefficients and also the extinction coefficients on each night. The observations were mostly done at five equal intervals of airmass in the range 1-2. Typical integration times were around 5 minutes each in $B$ and $\mathrm{H} \alpha, 2$ minutes in $V$ and 1 minute in $R$ band. The $\mathrm{H} \alpha$ filter used is an interference filter of bandwidth $160 \AA$ centred around $6563 \AA$. The images obtained using this filter are intended for studying the colour dependence of $\mathrm{H} \alpha$ to $R$ band flux ratios, of objects without line emission at $\mathrm{H} \alpha$. All the observations were done at the default gain (parameter cgain $=0$ ), which corresponds to 27.7 electrons/ADU (Prabhu, Mayya \& Anupama 1991). Throughout the observations, the temperature was stable at its normal operating value of $-120.2^{\circ} \mathrm{C}$ within two-tenths of a degree. Twilight skies were exposed in all the filters for purposes of flat fielding the images.

\section{Data analysis and results}

An inspection of many bias frames showed that the first 2-3 rows have lower counts and first 2-3 columns have higher counts compared to the mean value in the frame. Hence the first four rows and columns were trimmed out from each frame, bringing the frame size to $380 \times 572$ format. The trimmed bias frames did not show any pattern and hence mean values of bias frames were used for subtracting the bias levels from all the images. This mean value is found to decrease over a night by about 5-6 counts, in spite of a stable CCD temperature. This is depicted in Fig. 1 for four nights in February and March when the system was continuously on throughout the night (12-14 hours). Such an effect can be explained if some of the components in the 


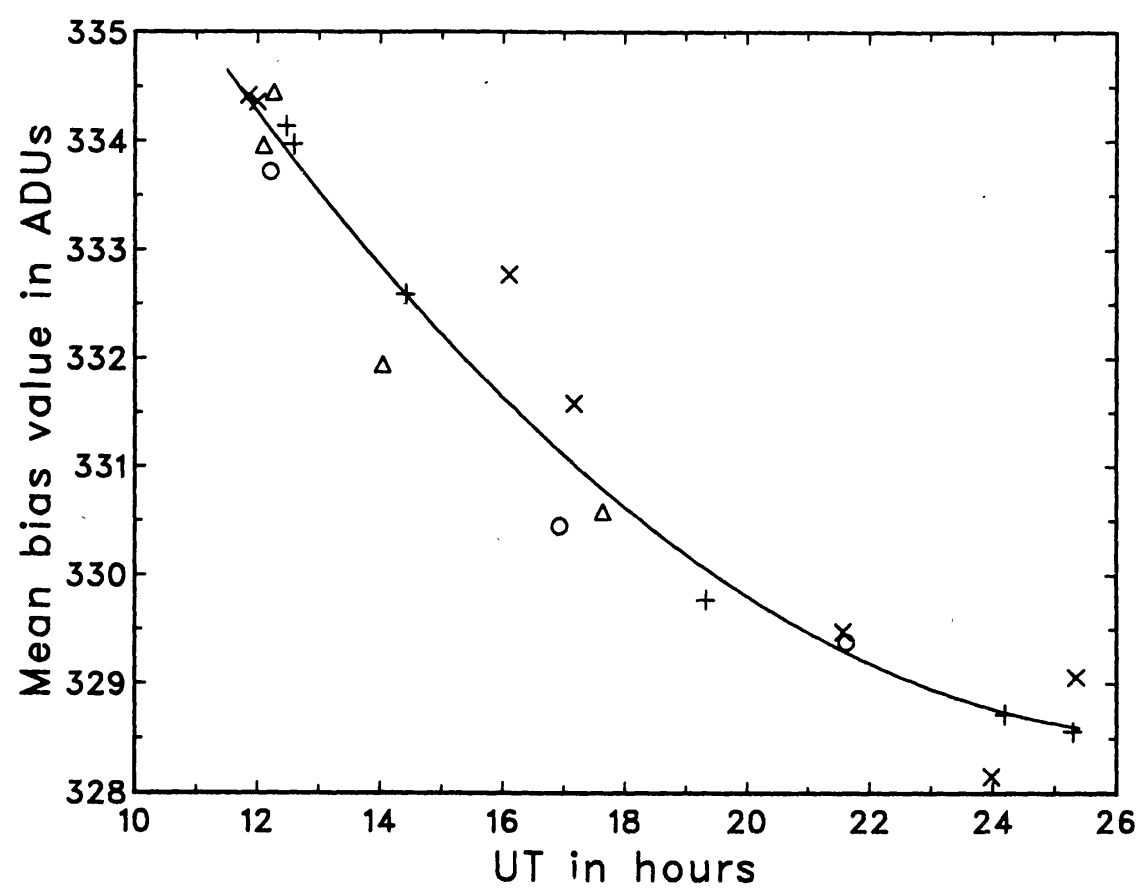

Figure 1. Variation of mean bias level of CCD over four different nights. The continuous line corresponds to a quadratic fit to the data. The first bias frame is obtained about $30 \mathrm{~min}$ after switching on the system. The CCD display temperature was stable at $-120.2^{\circ} \mathrm{C}$ throughout the nights.

controller are sensitive to ambient temperature, which is found to decrease steadily over a night. While subtracting bias levels from all object frames, this variation is taken into consideration. As can be seen in the figure, the decrease is more rapid in the early part compared to the later part.

Twilight sky flats exposed to a level of 10000-15000 counts in each filter are used in flat fielding the images. These count levels correspond to $2.7-4.1 \times 10^{5}$ electrons pixel $^{-1}$ or signal-to-noise $(\mathrm{S} / \mathrm{N})$ ratios of 500-600. Normally 2-3 flat exposures taken just after sunset and before sunrise are stacked together, normalized to the mean of a $10 \times 10$ box near the centre of the chip, and used for flat fielding the object frames taken during the night. Main features noticeable on all flat fields are:

i) A slow gradient from row number 200 to 1 , with the efficiency of pixels in the first row being around 80 per cent of those in the 200th row. Part of this might be due to vignetting since the filters employed had a small size $(25 \mathrm{~mm})$. Flat fielding procedure eliminates this gradient leaving residuals of only $\sim 2$ per cent.

ii) Dark rings of diameter 30-50 pixels. The annular portion of the ring is around 7 pixels wide with the darkest regions having efficiencies in the range 96-97 per cent. Such rings are reported in a similar system at University of Toronto Southern Observatory (UTSO), and are possibly due to the dust settled on the window of the CCD dewar (McCall, English \& Shelton 1989). Generally rings are well-corrected in the process of flat fielding. In addition there are about 10 highly inefficient pixels which are not well-corrected even after flat fielding.

Most of the above operations are performed using STARLINK software at the VAX 11/780 installation at VBO. 


\subsection{Magnitude Extraction}

The extraction of magnitudes from CCD images is carried out through software techniques. This can be done either by fitting 2-d profiles to stellar images or by simply summing all the counts within a reasonable software aperture centred around the stellar images. Howell (1989) describes an optimal extraction algorithm, based on the latter method. This method considers the magnitude and $\mathrm{S} / \mathrm{N}$ profiles, within a series of concentric apertures around stars. $\mathrm{S} / \mathrm{N}$ increases first because of increasing signal but begins to drop beyond a radius, where sky counts start dominating the weak wings of the stellar profiles. Magnitudes are extracted only up to a radius where $\mathrm{S} / \mathrm{N}$ peaks, and to account for the flux contained beyond this radius, a correction factor is applied based on well-exposed stars in the frame. This method reduces the errors introduced by inaccurate sky subtraction. A computer programme based on this method is developed locally under the STARLINK environment and is used for the extraction of all stellar magnitudes for this work. In all our frames stars brighter than $13 \mathrm{mag}$ are well exposed with $\mathrm{S} / \mathrm{N}$ peaking between 5-15 pixels and hence 'aperture magnitudes' at fixed apertures (say 20 pixel $=7$ arcsec radius), give values within 0.01 magnitudes of those derived from optimal extraction method. Growth curves and $\mathrm{S} / \mathrm{N}$ plots for some of the stars in a frame are shown in Fig. 2.

\subsection{Atmospheric Extinction}

The brightest two stars in the dipper asterism field were used for atmospheric extinction determinations. These stars have a large colour difference $(\Delta(B-V)=1.4 \mathrm{mag})$ and hence are useful in determining the colour dependence of extinction coefficients. The equations discussed by Hardie (1962) are used in the determination of extinction coefficient and are as follows:

$$
\begin{aligned}
v & =v_{0}+k_{v} X, \\
b-v & =(b-v)_{0}+k_{b-v} X, \\
v-r & =(v-r)_{0}+k_{v-r} X, \\
k_{v} & =k_{v}^{\prime}-k_{v}^{\prime \prime}(b-v), \\
k_{b-v} & =k_{b-v}^{\prime}-k_{b-v}^{\prime \prime}(b-v), \\
k_{v-r} & =k_{v-r}^{\prime}-k_{v-r}^{\prime \prime}(v-r),
\end{aligned}
$$

where the lower case letters $b, v$ and $r$ stand for instrumental magnitudes in $B, V$ and $R$ filters respectively and the suffix 0 denotes the 'above atmosphere' instrumental magnitudes. $k^{\prime}$ and $k^{\prime \prime}$ with suffixes represent first-order and second-order extinction coefficients respectively.

Equations (1) and (2) were solved to determine $k^{\prime \prime}$ first and then $k^{\prime}$ values. The second-order extinction coefficients obtained on different nights were found to be similar. The mean values of these coefficients are 0.010 in $v, 0.026$ in $b-v$ and 0.010 in $v-r$. These mean values are used in the determination of first-order coefficients. The values of first-order coefficients for eight photometric nights appear in Table 2 together with their errors. The last line in the table contains the mean values with the errors representing the nightly variations of the coefficients. The corresponding 

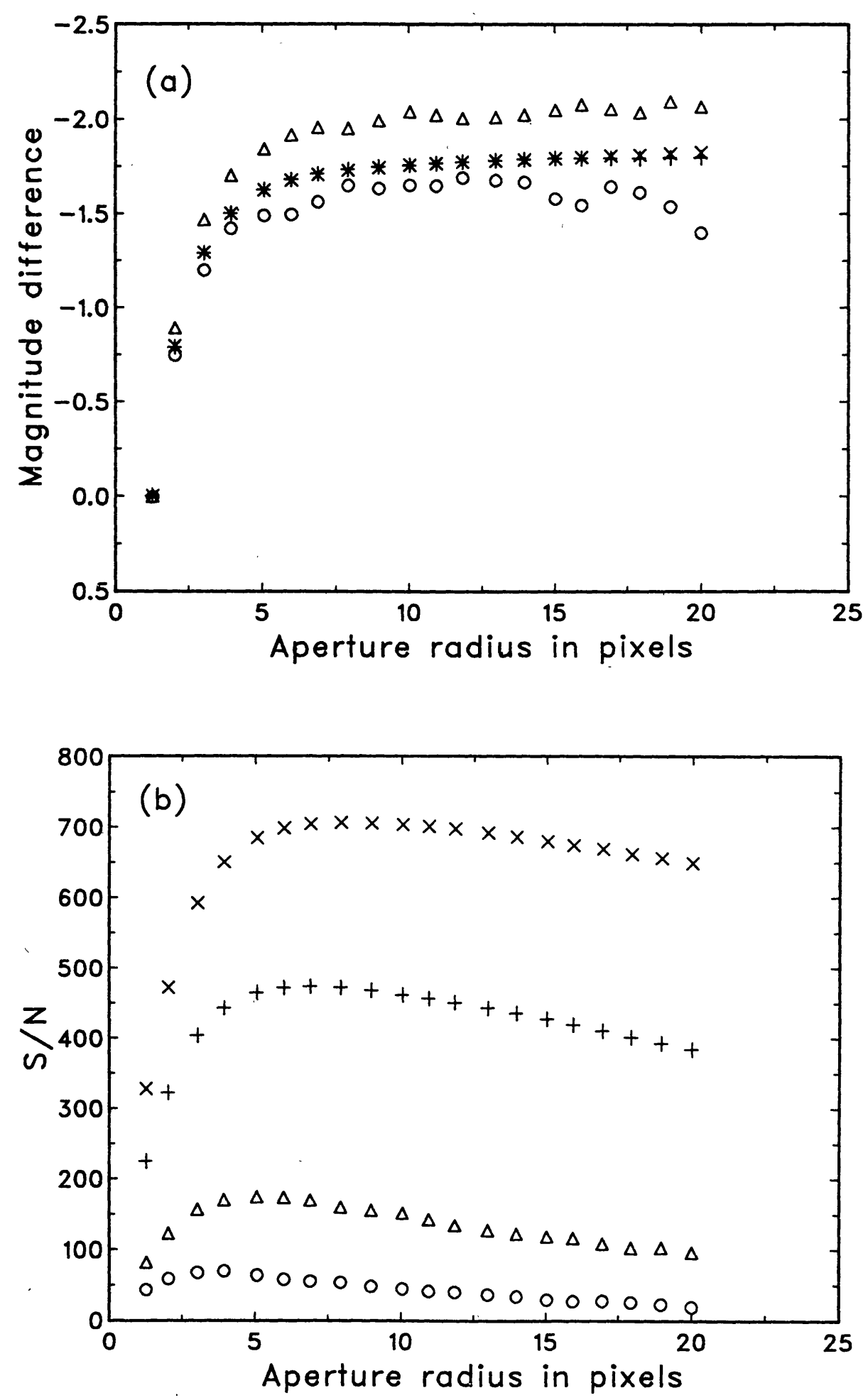

Figure 2. (a) CCD aperture growth curves, and (b) $S / N$ ratio, for four stars in a single CCD frame. The magnitudes of these stars as estimated from optimal extraction method are $12.37(x)$, $13.20(+), 15.04(\Delta)$ and $16.33(O)$ in $V$ band. For the sake of display, magnitude within an aperture of 1 pixel radius is subtracted from all concentric apertures for all the stars. Note that the growth curves for the fainter two stars deviate from the others due to an underestimation $(\Delta)$ or overestimation $(O)$ of sky. The $\mathrm{S} / \mathrm{N}$ peaks at a smaller radius for fainter stars. 
Table 2. First-order atmospheric extinction coefficients.

\begin{tabular}{lccc}
\hline Date & $k_{\mathrm{b}}^{\prime}$ & $k_{\mathrm{v}}^{\prime}$ & $k_{\mathrm{r}}^{\prime}$ \\
\hline 1991 & & & \\
Jan 13 & $0.341 \pm 0.023$ & $0.166 \pm 0.015$ & $0.090 \pm 0.013$ \\
Feb 13 & $0.407 \pm 0.003$ & $0.214 \pm 0.006$ & $0.113 \pm 0.002$ \\
Feb 14 & $0.385 \pm 0.002$ & $0.206 \pm 0.001$ & $0.119 \pm 0.002$ \\
Feb 15 & $0.472 \pm 0.011$ & $0.295 \pm 0.012$ & $0.212 \pm 0.016$ \\
Mar 16 & $0.397 \pm 0.006$ & $0.187 \pm 0.002$ & $0.102 \pm 0.002$ \\
Mar 17 & $0.430 \pm 0.003$ & $0.224 \pm 0.003$ & $0.133 \pm 0.007$ \\
Mar 18 & $0.478 \pm 0.005$ & $0.259 \pm 0.003$ & $0.145 \pm 0.002$ \\
Apr 14 & $0.418 \pm 0.008$ & $0.237 \pm 0.007$ & $0.153 \pm 0.006$ \\
Mean & $0.416 \pm 0.045$ & $0.224 \pm 0.041$ & $0.133 \pm 0.038$ \\
\hline
\end{tabular}

values for the colours are $0.193 \pm 0.017$ in $b-v$ and $0.090 \pm 0.012$ in $v-r$. Allowing for the fact that we have used instrumental colours in our extinction equations, the $B-V$ coefficient reduces to 0.167 . Theoretical values for the site calculated using the equations of Bessell (1990) are 0.213, 0.135 and 0.093 for $V, B-V$ and $V-R$ respectively. The relatively higher values for the extinction are because of the lower altitude of the site $(725 \mathrm{~m})$. Thus the agreement between the observed and theoretical values are good for $V$ and $V-R$ while the observed coefficients are $\sim 0.03$ mag higher for $B-V$.

\subsection{Photometric Transformations}

The above atmosphere magnitudes and colours $v_{0},(b-v)_{0}$ and $(v-r)_{0}$ are transformed into the Johnson-Cousins $B V R$ system by fitting the equations of the following form to our data.

$$
\begin{aligned}
& B-V=\alpha_{b-v}+\beta_{b-v}(b-v)_{0}, \\
& V-R=\alpha_{v-r}+\beta_{v-r}(v-r)_{0}, \\
& V-v_{0}=\alpha_{v}+\beta_{v}(b-v)_{0}, \\
& B-b_{0}=\alpha_{b}+\beta_{b}(b-v)_{0}, \\
& R-r_{0}=\alpha_{r}+\beta_{r}(v-r)_{0}
\end{aligned}
$$

where the upper-case letters $B, V$ and $R$ are standard magnitudes in the corresponding filters, taken from Chevalier \& Ilovaisky (1991). The fits to the first three equations are shown in Figs 3(a)-3(c), with the ordinates representing colour and magnitude differences. Similar transformations were tried with Schild's (1983) data and we confirm the finding of Chevalier \& Ilovaisky that the zero points for the brightest 2 stars are different from the rest in Schild's data. The transformation coefficients are derived for all the nights. Colour coefficients $(\beta)$ obtained on different nights agree well while the zero points $(\alpha)$ differ by as much as 0.1 mag on different nights. In Table 3 , we list the transformation coefficients obtained for 1991 April 14, together with the standard errors of coefficients and the standard error of the fit. The transformations involving 
Photometric calibration of CCD camera
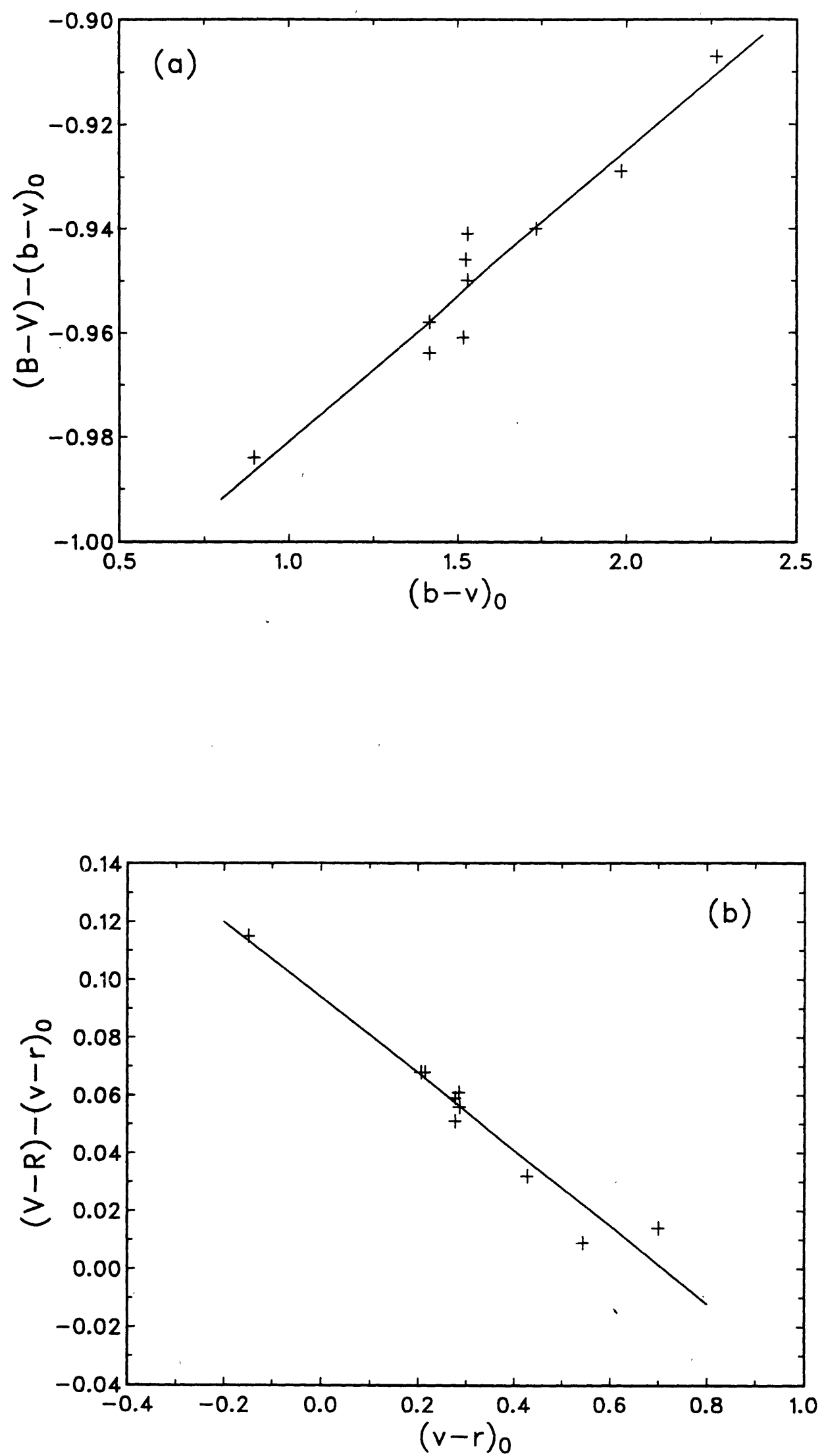

Figure 3. 

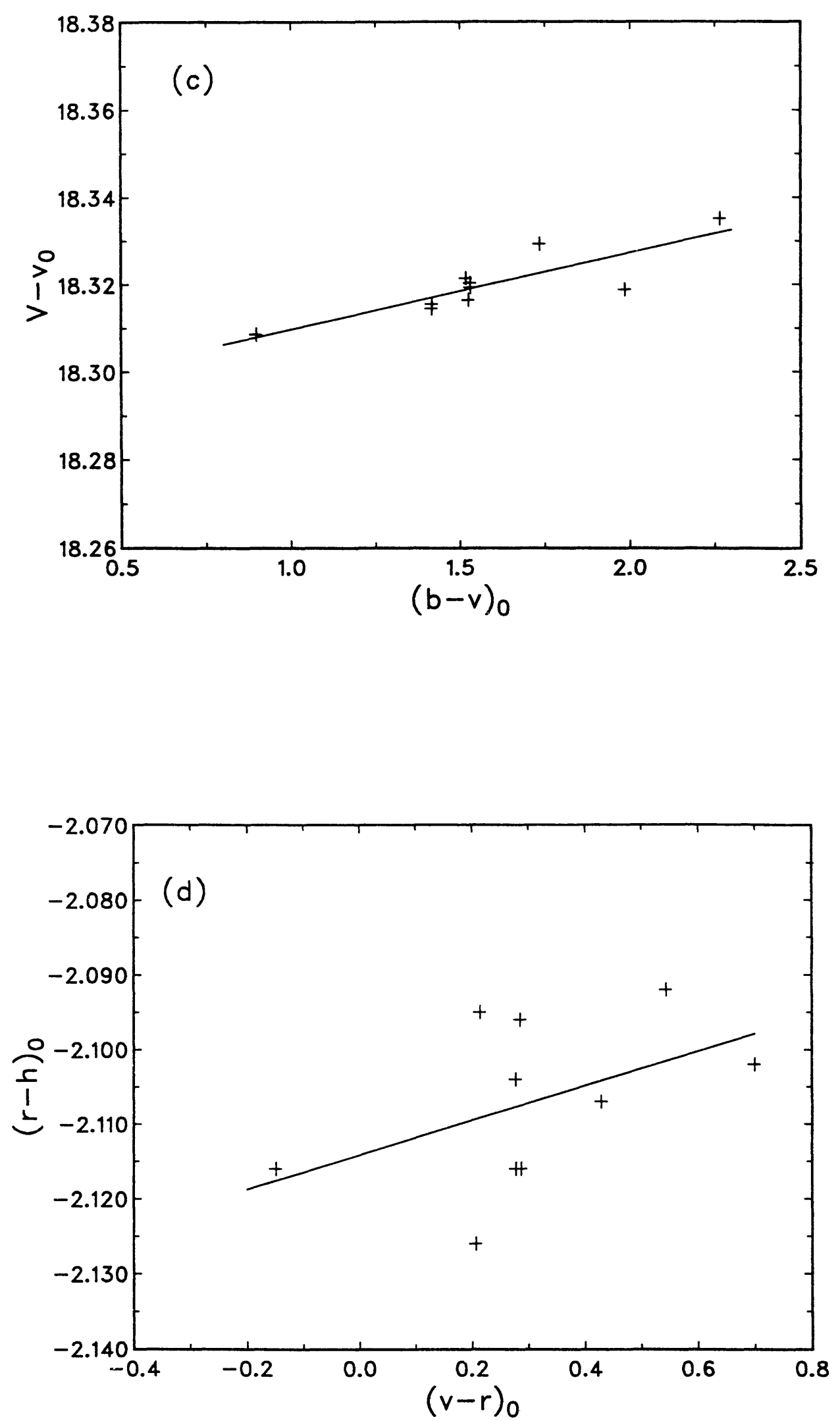

Figure 3. Standard - instrumental colours (a) $B-V$, and (b) $V-R$, and magnitude differences (c) $\left(V-v_{0}\right)$, and (d) $(r-h)_{0}$ plotted against the extinction-corrected instrumental colours. The least squares fit to the data is represented by the line. 
Table 3. Transformation coefficients to Johnson-Cousins system.

\begin{tabular}{lrcc}
\hline Quantity & \multicolumn{1}{c}{$\alpha$} & $\beta$ & Std. error \\
\hline$B-V$ & $-1.037 \pm 0.006$ & $1.056 \pm 0.004$ & 0.006 \\
$V-R$ & $0.094 \pm 0.003$ & $0.868 \pm 0.007$ & 0.007 \\
$V$ & $18.292 \pm 0.004$ & $0.018 \pm 0.003$ & 0.004 \\
$B$ & $17.256 \pm 0.007$ & $0.073 \pm 0.005$ & 0.007 \\
$R$ & $18.217 \pm 0.003$ & $0.160 \pm 0.007$ & 0.007 \\
$(r-h)^{\mathrm{a}}$ & $-2.114 \pm 0.004$ & $0.023 \pm 0.011$ & 0.011 \\
\hline
\end{tabular}

a Transformation of instrumental ' $\mathrm{H} \alpha$ magnitudes' to instrumental $r$ magnitude (see text for details).

$R$ are more colour-dependent than the rest, which is expected because the effective wavelength of our $R$ band is around $300 \AA$ redder than the standard.

\section{$3.4 \mathrm{H} \alpha$ Photometry}

The accuracy of emission-line photometry largely depends on the estimation of the contribution of the in-band continuum. Obtaining well-exposed continuum (off-band) images with filters of bandwidth $<200 \AA$, using telescopes of $1-\mathrm{m}$ class, is difficult and costly in terms of telescope time. This leads to subtraction of poorly exposed continuum images from well-exposed line images, when continuum filters of similar widths as those of line filters are used. Most of the uncertainties involved in continuum subtraction can be eliminated if the in-band continuum can be estimated from an image taken with a broader filter. However, this might lead to additional errors depending on the spectrum of the emitting object near the line of interest. In order to study these errors, we observed the dipper asterism field also with the $\mathrm{H} \alpha$ filter on most of the nights.

The $\mathrm{H} \alpha$ filter images of stars without strong $\mathrm{H} \alpha$ emission or absorption line will have contribution mainly from the continuum. The relative bandwidth of the $\mathrm{H} \alpha$ filter with respect to the $R$ band can hence be obtained by transforming the data obtained through the $\mathrm{H} \alpha$ and $R$ filters over a wide range of colours. For this purpose we define an 'instrumental $\mathrm{H} \alpha$ magnitude' $h_{0}$ in analogy with broad continuum bands. The magnitude difference $(r-h)_{0}$ for stars are fitted against their $(v-r)_{0}$ colour with an equation of the form

$$
(r-h)_{0}=\alpha_{r-h}+\beta_{r-h}(v-r)_{0} .
$$

The resulting coefficients $\alpha_{r-h}$ and $\beta_{r-h}$ are given in Table 3 for one of the nights, and the fit is shown in Fig. 3(d). Neglecting the small colour term, the zero point $\alpha_{r-h}$ corresponds to a factor of 7.09 between the broadband $R$ filter and the narrowband $\mathrm{H} \alpha$ filter. Variation of this ratio remained within 3 per cent on all the nights. Scaling the $R$ band fluxes with this ratio would thus give a good estimation of the in-band continuum in $\mathrm{H} \alpha$ over a wide range of colours. Waller (1990) has demonstrated this by obtaining continuum subtracted $\mathrm{H} \alpha$ images and line subtracted continuum images of Orion nebula, from observations in $\mathrm{H} \alpha$ and $R$ bands. We also transformed our instrumental $\mathrm{H} \alpha$ magnitudes to the $R$ band, in order to flux calibrate $\mathrm{H} \alpha$ images. 
This leads to a value of $10^{-12.99} \mathrm{erg} \mathrm{cm}^{-2} \mathrm{~s}^{-1}$ of incident flux for 1 count $\mathrm{s}^{-1}$ detected in the $\mathrm{H} \alpha$ filter. The conversion factor is also estimated by observing the spectrophotometric standard stars EG 99 and HD 60778. The standard spectrum of the star is multiplied by the transmission curve of the filter and integrated to obtain the total $\mathrm{H} \alpha$ flux expected within the band. The conversion factors derived from these two stars were $10^{-13.02}$ and $10^{-13.01} \mathrm{erg} \mathrm{cm}^{-2} \mathrm{~s}^{-1}$ for 1 count $\mathrm{s}^{-1}$ respectively.

\subsection{Photometric Repeatability and Sensitivity}

The availability of a large number of frames allowed us to estimate the internal consistency of our magnitude measurements. All the stars in a given frame are first transformed into the standard system using the zero points derived from one of the stars in the field (stars F81). All such measurements on a single night (17 March) were used to obtain the average magnitude and rms error for each star given in Table 4. See Chevalier \& Ilovaisky (1991) for identification numbers and chart. The rms errors of magnitude and colour determination are $\sim 0.01$ mag for majority of the stars in the field. The derived magnitudes and colours themselves agree well with the standard values within $0.01 \mathrm{mag}$. On the other hand, the zero points on different nights vary by as much as $0.1 \mathrm{mag}$ and hence by obtaining zero points for each night, it is possible to reach an overall photometric accuracy of $0.01 \mathrm{mag}$.

Five of the 16 stars observed exhibited an appreciable departure $(\sim 0.03-0.05 \mathrm{mag})$ from Chevalier \& Ilovaisky (1991)-F117, F134, F135, I-9 and I-227. Of these, F134, F135 and I-9 have higher rms errors and are spatially together on the CCD frame, occupying the first 100 rows. I-227 is the faintest in our list. Hence the observed difference may not be significant for these stars. On the other hand F117 was

Table 4. Derived magnitudes and colours of M67 stars.

\begin{tabular}{lrrcrrrr}
\hline Ident. & $V$ & \multicolumn{1}{c}{$\Delta^{\mathrm{a}}$} & \multicolumn{1}{c}{$B-V$} & \multicolumn{1}{c}{$\Delta^{\mathrm{a}}$} & \multicolumn{1}{c}{$V-R$} & $\Delta^{\mathrm{a}}$ & $\mathrm{N}$ \\
\hline F81 & $10.022 \pm 0.000$ & 0.000 & $-0.086 \pm 0.000$ & 0.000 & $-0.034 \pm 0.000$ & 0.000 & 5 \\
F108 & $9.713 \pm 0.005$ & -0.011 & $1.353 \pm 0.005$ & 0.006 & $0.734 \pm 0.004$ & -0.020 & 5 \\
F117 & $12.602 \pm 0.004$ & 0.034 & $0.790 \pm 0.013$ & 0.004 & $0.479 \pm 0.003$ & -0.018 & 5 \\
F124 & $12.121 \pm 0.002$ & 0.003 & $0.464 \pm 0.008$ & -0.006 & $0.294 \pm 0.005$ & -0.011 & 5 \\
F127 & $12.764 \pm 0.004$ & 0.001 & $0.555 \pm 0.009$ & 0.002 & $0.349 \pm 0.005$ & -0.020 & 5 \\
F134 & $12.209 \pm 0.023$ & 0.038 & $0.580 \pm 0.009$ & -0.001 & $0.357 \pm 0.003$ & -0.010 & 5 \\
F135 & $11.383 \pm 0.025$ & 0.049 & $1.066 \pm 0.011$ & -0.009 & $0.595 \pm 0.005$ & -0.022 & 5 \\
F130 & $12.874 \pm 0.011$ & 0.010 & $0.453 \pm 0.012$ & 0.000 & $0.286 \pm 0.006$ & -0.010 & 5 \\
F128 & $13.132 \pm 0.008$ & 0.015 & $0.594 \pm 0.021$ & -0.014 & $0.345 \pm 0.012$ & -0.008 & 5 \\
F129 & $13.166 \pm 0.014$ & 0.007 & $0.600 \pm 0.014$ & -0.011 & $0.359 \pm 0.002$ & -0.016 & 5 \\
I-228 & $12.400 \pm 0.002$ & -0.003 & $0.744 \pm 0.004$ & 0.000 & $0.434 \pm 0.000$ & -0.008 & 2 \\
F111 & $12.733 \pm 0.002$ & 0.009 & $0.564 \pm 0.010$ & 0.004 & $0.350 \pm 0.004$ & -0.014 & 2 \\
I-9 & $13.144 \pm 0.041$ & 0.048 & $0.591 \pm 0.010$ & -0.007 & $0.351 \pm 0.006$ & -0.016 & 2 \\
F83 & $13.212 \pm 0.003$ & -0.013 & $0.586 \pm 0.018$ & 0.012 & $0.358 \pm 0.003$ & -0.007 & 2 \\
I-227 & $14.158 \pm 0.003$ & -0.026 & $0.764 \pm 0.001$ & -0.057 & $0.414 \pm 0.008$ & 0.002 & 2 \\
I-226 & $13.940 \pm 0.004$ & -0.007 & $0.623 \pm 0.007$ & -0.031 & $0.356 \pm 0.009$ & -0.004 & 2 \\
\hline
\end{tabular}

a The differences in magnitudes and colours in the sense Chevalier \& Ilovaisky (1991) - present work 
Table 5. Filter properties and the sensitivity of the system.

\begin{tabular}{|c|c|c|c|c|c|c|}
\hline Sl. No. & Quantity & $B$ & $\boldsymbol{V}$ & $\boldsymbol{R}$ & $\mathbf{H}_{\alpha}$ & Units \\
\hline 1 & $\hat{i}_{0}$ & 4351. & 5419. & 6713. & 6567. & $\AA$ \\
\hline 2 & $\Delta i$ & 965. & 920. & 975. & 160. & $\AA$ \\
\hline 3 & $\mathrm{~F}_{\lambda}(0)$ & 6.90 & 3.83 & 1.81 & 2.04 & $10^{-9} \mathrm{erg} \mathrm{cm}^{-2} \mathrm{~s}^{-1} \AA^{-1}$ \\
\hline 4 & $-\mathrm{m}_{\mathrm{th}}=2.5 \log S_{0}$ & 21.36 & 20.96 & 20.72 & 18.61 & $\mathrm{mag}$ \\
\hline 5 & $-\mathrm{m}_{i}$ & 17.22 & 18.29 & 18.22 & 16.11 & $\mathrm{mag}$ \\
\hline 6 & $\eta$ & 2.04 & 8.33 & 9.68 & 10.18 & $\%$ \\
\hline \multirow[t]{2}{*}{7} & $S_{0}(\lambda)(m=15) X=0$ & 7.73 & 20.70 & 19.41 & 2.78 & count $\mathrm{s}^{-1}$ \\
\hline & estimate $X=1$ & 5.60 & 17.22 & 17.54 & 2.51 & count $\mathrm{s}^{-1}$ \\
\hline 8 & $\mu_{i}$ & 14.98 & 16.05 & 15.98 & & $\operatorname{mag} \operatorname{arcsec}^{-2}$ \\
\hline 9 & $\mu_{s k y}$ & 21.43 & 20.62 & 19.62 & & $\mathrm{mag} \operatorname{arcsec}^{-2}$ \\
\hline 10 & Flux & & & & 1.00 & $10^{-13} \mathrm{erg} \mathrm{cm}^{-2} \mathrm{~s}^{-1}$ \\
\hline 11 & Sky brightness & & & & 2.02 & $\begin{array}{l}10^{-15} \mathrm{erg} \mathrm{cm}{ }^{-2} \mathrm{~s}^{-1} \\
\operatorname{arcsec}^{-2}\end{array}$ \\
\hline
\end{tabular}

Notes: See text following Equation (6) for the explanation of quantities in row numbers 1-3. $S_{0}$ appearing in row 4 is calculated using Equation (6) with $\eta=1$ and $m=0$. In row 5 zero points of the transformation equations are given, which indicate the magnitude which would produce a count rate of 1 count $\mathrm{s}^{-1} . \eta$ values given in row 6 are the derived efficiencies for the telescope + filter + CCD combination. In row 7 estimated count rates for a star of magnitude 15 are given at airmasses 0 and 1 . Row 8 lists the surface brightness required to produce a count rate of 1 count s $^{-1}$ pixel $^{-1}$ and row 9 gives the surface brightness of the sky close to the zenith as observed on $16 \mathrm{Mar} 1991$ which was a moon-less night. $\mathrm{H} \alpha$ line flux required to produce 1 count $\mathrm{s}^{-1}$ is given in row 10 . Row 11 contains the sky surface brightness in flux units within $\mathrm{H} \alpha$ filter for the same night as for other filters.

consistently brighter by $0.03 \mathrm{mag}$ on all the three nights in March. The magnitudes of this star on other nights are 12.645 (13 Jan), 12.631 (13 Feb), 12.626 (14 Feb), 12.608 (16 Mar), 12.606 (18 Mar) and 12.636 (14 Apr). This star is possibly a small amplitude variable and more observations are required to determine its light curve.

The overall efficiency of the system in $B V R H \alpha$ bands is estimated by comparing the zero points in the transformation equations with the expected values. The expected counts for a star of magnitude $m$ using a telescope of diameter $D$ is given by McLean (1989),

$$
\frac{S_{0}}{1 \text { count s}^{-1}}=\frac{3.95 \times 10^{11}}{g}\left(\frac{D}{1 \mathrm{~m}}\right)^{2}\left(\frac{\lambda}{\AA}\right)\left(\frac{\Delta \lambda}{\AA}\right) F_{\lambda}(0) 10^{-0.4 \mathrm{~m}} \varepsilon \eta(\lambda),
$$

where $g$ in electrons count ${ }^{-1}$ is the system gain (27.7 in the present case), $\lambda, \Delta \lambda$ are respectively the effective wavelength and bandwidth of the filters, as computed based on the transmission curves for Schott glass filters, $F_{\lambda}(0)$ is the flux density in erg $\mathrm{cm}^{-2} \mathrm{~s}^{-1} \AA^{-1}$ from a star of magnitude 0 at the wavelength $\lambda$ above the earth's atmosphere, $\varepsilon$ is the fractional reflecting area of the mirror surface $(\sim 0.85)$ and $\eta$ is the efficiency of the system (mirrors + filter + CCD). The resulting values of $\eta$ are given in Table 5. The peak efficiency is around 10 per cent and lies in the red region. In the blue region the efficiency drops to just 2 per cent. It was not possible to investigate the causes of this poor response in the blue. 


\subsection{Dynamic Range and Linearity}

The effective dynamic range of a CCD camera is set by the ratio of the maximum source signal readable to the readout noise or the least count, whichever is larger. In our system, the maximum readable signal is determined by the A/D converter rather than the full-well capacity of the CCD. The expected readout noise for the system is around 7-8 electrons, which is smaller than 1 count (27.7 electrons) at default gain. Thus, after accounting for the bias level, the available dynamic range is about 16000 or $10.5 \mathrm{mag}$. The requirement on the accuracy at the faintest level will reduce this range to a smaller value. We could detect $17 \mathrm{mag}$ stars in one minute integration in $R$ band when the brightest star in the field $(9.7 \mathrm{mag})$ was close to saturation.

The maximum readable signal is about 445000 electrons, which is slightly less than the full well capacity of 500000 electrons for the chip. Thus, the nonlinear responses if any, may be evident at the highest count levels. To check the linearity, we compared the peak counts of stars in a single frame of M67. For a frame with peak counts around 11000 , the peak counts for individual stars were compared with the magnitude of the star. The resulting data is fitted with a straight line giving rise to the following equation,

$$
-2.5 \log R_{\text {peak }}=(1.014 \pm 0.004) R-19.21 \pm 0.02,
$$

where $R_{\text {peak }}$ is the observed peak counts in the $R$ band for a star of red magnitude $R$. Thus the system appears linear to $\sim 1$ per cent in the range 300-11000 counts (8200-300000 electrons). Similar conclusions were drawn by McCall, English \& Shelton (1989) for the UTSO system.

\section{Conclusions}

CCD observations of the dipper asterism stars in M67 with glass filter combinations allowed us to obtain good transformations equations to the standard $B V R$ system. These transformations are limited to the range $-0.09<B-V<1.35$ and $-0.03<$ $V-R<0.73$ due to the availability of bright standard stars in the field of M67 used. It is desirable to extend the work to a larger range in colour. The relative $B V R$ magnitudes of stars within a frame are accurate to within 0.01 mag and the accuracy of photometry is mainly determined by extinction and zero point determinations. The in-band continuum in $\mathrm{H} \alpha$ filter of $160 \AA$ width can be estimated from $R$ band images to within 3 per cent accuracy. The efficiency of the telescope-filter-CCD combination peaks in the $R$ band reaching values around 9.7 per cent. The efficiency drops to 8.3 per cent in the $V$ band and to 2.0 per cent in the $B$ band. The dynamic range of the system with default gain setting corresponding to 27.7 electrons count ${ }^{-1}$ is 16000 and the system is linear to 1 per cent accuracy upto 11000 counts. The star F117 appears to be a small-amplitude $(\sim 0.05 \mathrm{mag})$ variable.

\section{Acknowledgements}

I would like to thank T. P. Prabhu for the guidance rendered by him throughout this work and also for reading the manuscript. I am also thankful to S. Mohin for 
sharing with me his vast experience in photoelectric photometry. The filters were cut and ground to the required thickness and sizes at the optics laboratory of the Institute, for which I thank A. K. Saxena and J. P. Lancelot. I thank A. K. Pati for installation and supervision of the CCD system, and K. Kuppuswamy, M. Appakutty and A. Muniyandi for their assistance during observations. Finally, I wish to thank the referees M. S. Bessell and I. S. McLean for their valuable comments.

\section{References}

Bessell, M. S. 1976, Publ. astr. Soc. Pacific, 88, 557.

Bessell, M. S. 1990, Publ. astr. Soc. Pacific, 102, 1181.

Chevalier, C., Ilovaisky, S. A. 1991, Astr. Astrophys. Suppl., 90, 225.

Fernie, J. D. 1974, Publ. astr. Soc. Pacific, 86, 837.

Gilliland, R. L., Brown, T. M., Duncan, D. K., Suntzeff, N. B., Lockwood, G. W., Thompson, D. T., Schild, R. E., Jeffrey, W. A., Penprase, B. E. 1991, Astr. J., 101, 541.

Hardie, R. H. 1962, in Astronomical Techniques, Ed. W. A. Hiltner, Univ. Chicago Press, p. 178. Howell, S. B. 1989, Publ. astr. Soc. Pacific, 101, 616.

Joner, M. D., Taylor, B. J. 1990, Publ. astr. Soc. Pacific, 102, 1004.

McCall, M. L., English, J., Shelton, I. 1989, J. R. astr. Soc. Canada, 83, 179.

McLean, I. S., 1989, in Electronic and Computer-Aided Astronomy, Ellis Horwood, Chichester.

Prabhu, T. P., Mayya, Y. D., Anupama, G. C. 1991, Preprint.

Sagar, R., Pati, A. K. 1989, Bull. astr. Soc. India, 17, 6.

Schild, R. E. 1983, Publ. astr. Soc. Pacific, 95, 1021.

Waller, W. H. 1990, Publ. astr. Soc. Pacific, 102, 1217. 